University for Business and Technology in Kosovo

UBT Knowledge Center

UBT International Conference

2016 UBT International Conference

Oct 28th, 9:00 AM - Oct 30th, 5:00 PM

\title{
Why Modeling Complex Dynamic Systems using Fuzzy Cognitive Maps?
}

Peter P. Groumpos

University of Patras, groumpos@ece.upatras.gr

Follow this and additional works at: https://knowledgecenter.ubt-uni.net/conference

Part of the Engineering Commons, and the Physical Sciences and Mathematics Commons

\section{Recommended Citation}

Groumpos, Peter P., "Why Modeling Complex Dynamic Systems using Fuzzy Cognitive Maps?" (2016). UBT International Conference. 47.

https://knowledgecenter.ubt-uni.net/conference/2016/all-events/47

This Event is brought to you for free and open access by the Publication and Journals at UBT Knowledge Center. It has been accepted for inclusion in UBT International Conference by an authorized administrator of UBT Knowledge Center. For more information, please contact knowledge.center@ubt-uni.net. 
Book of Proceedings

International Conference on Mechatronics, Sciences in Energy Efficiency Engineering,

System Engineering and Robotics

\title{
Why Modeling Complex Dynamic Systems using Fuzzy Cognitive Maps?
}

\author{
Peter P. Groumpos \\ Laboratory for Automation and Robotics, University of Patras, Department of Electrical \\ and Computer Engineering, Rion 26500, Patras, Greece \\ groumpos@ece.upatras.gr
}

\begin{abstract}
The difficult problem of modeling Complex Dynamic Systems (CDS) is carefully reviewed. Main characteristics of CDS are considered and analyzed. Today's mathematical models and approaches cannot provide satisfactory answers to the challenging problems of the society. The key problem of complex dynamic systems and control theory consists in the development of methods of qualitative analysis of the dynamics and behavior of such systems and in the construction of efficient control algorithms for their efficient operation. The purpose of control to bring the system to a point of its phase space which corresponds to maximal or minimal value of the chosen efficiency criterion is reviewed and analyzed. The reasons for using Fuzzy Cognitive Maps (FCMs) in modeling Complex dynamic Systems are provided. The basics of FCMs are briefly presented. An illustrative example is considered and interesting results are presented and discussed.
\end{abstract}

Keywords: Modelling, Complex dynamic systems, Fuzzy logic, Intelligent Systems, Fuzzy Cognitive Maps

\section{Introduction}

Today one scientific practice that all of us must be doing constantly is to listen to others and raise serious and challenging questions. Here are some. What is a Complex Dynamic System (CDS)? What are its main characteristics? What are the best models for studying them? Do all models have detailed software tools that can adequately simulate their behavior? Do we have a clear and sound scientific understanding of the concepts of chaos, complexity and uncertainty? And how these three concepts are taken into consideration when studying, modeling, analyzing and designing a CDS?? How theories of Large Scale Systems (LSS) as well as for Multilevel Hierarchical have taken into consideration the concepts of chaos, complexity and uncertainty? We can continue raising one question after the other and then try to understand the provided solutions and then raising more questions. Do all these models and associated solutions provide satisfactory and working conditions to the everyday behavior of the complex dynamic systems? We can say that for a good number of real cases the provided models and solutions meet the objectives and goals of the complex dynamic system. However there are also a good and large number of situations where today's models and solutions fail to give satisfactory answers to a number of problems associated with them. 
Why Modeling Complex Dynamic Systems using Fuzzy Cognitive Maps?

Can we search and identify the sources for this failure? May be! I believe that the main reasons are our inability to comprehend and understand well and precisely the actual dynamic and chaotic behavior of complex dynamic systems in the presence of uncertainty, fuzziness and structural complexity. This is also due to the fact all these concepts have different interpretations and mathematical explanations by different people. Another important factor here is the solid knowledge and experience of the scientists been involved in the process and on making decisions subsequently. Some scientist combine all above factors into one term: UNCERTAINTY and try to explain everything using theories ant techniques that have been developed to model, understand, analyze and finally arrive in taking decisions. However all these efforts have still failed to provide satisfactory answers to the real problems faced by the behavior of complex dynamic systems?

\section{Challenging Issues in Modeling and Controlling Complex Dynamic Systems}

Modeling is a fundamental work which is always the starting point for control, optimisation, and implementation of complex dynamic systems (CDS). Complex dynamic systems present problems both in mathematical modelling and philosophical foundations. CDS comprise of collections of many heterogeneous entities which interact with other entities and their environment which usually are having a lot of uncertainties and structural complexities. Interactions among subsystems are localized, often seeking autonomy and self-organizing, while most of the times are nonlinear, dynamic, fuzzy and possibly chaotic. The study of CDS represents a new approach to science that investigates how relationships between parts give rise to the collective behaviors of a system and how the system interacts and forms relationships with its environment. CDS have some specific characteristics, among which are: uniqueness, weak structuredness of knowledge about the system, incompleteness of its dynamic behavior, antagonism among different agents, the composite nature of system, heterogeneity of elements composing the system. Furthermore decisions must be made ensuring the smooth, reliable, stable and cost effective operation of each of the subsystem as well the whole CDS [11],[12],[13]. Thus modeling CDSs is indeed a real chalenge. It is not so a straightforward and an easy task. Indeed it is a difficult exercise and cannot be completed by using today's formal methods.

Another important feature of CDS is that a network structure, including hierarchical one, selforganization can amount to: (1) disconnecting certain constituent nodes from the system, (2) connecting previously disconnected nodes to the same or to other nodes, (3) acquiring new nodes, (4) discarding existing nodes, (5) acquiring new links, (6) discarding existing links, (7) removing or modifying existing links. In addition CDS should have a number of properties-abilities such as; co-evolution, anticipation, adaptation, emergence, self-evaluation, Robustness and wisdom [1], [2], [5].

Finally collective dynamics of a CDS give rise to 'Emergent Evolution Properties' (EEP) at higher scales in space and/or time among some which are: cooperation such as swarming, intelligence, consciousness, genetic regulation - homeostasis, development, disease, cascading failures in electrical grid, invasiveness in plants, hurricanes and self-repairing materials. Under such conditions, the key problem of complex dynamic systems and control theory consists in the development of methods of qualitative analysis of the dynamics and behavior of such systems and in the construction of efficient control algorithms for their efficient operation. In a general case, the purpose of control is to bring the system to a point of its phase space which corresponds to maximal or minimal value of the chosen efficiency criterion. Another one of the main and actual problems in the theory of complex dynamical systems and control sciences is a solution of "ill-posed, weakly- and 
poorly-structured and weakly- formalizable complex problems" associated with complex technical, organizational, social, economic, cognitive and many other objects, and with the perspectives of their evolution. Therefore the modeling and analysis of complex dynamic systems in the presence of principally non- formalizable problems and not probable of having strict mathematical formulation of the system, on environments that decisions are semi- structured or unstructured, knowledge-base systems (KBS) needs to be readdressed. All above characteristics must be taken into consideration. Construction of models of CDS must be based on the use of experts and their extensive knowledge about the system. This knowledge should be wisely used. However qualitative description of most of the parameters of complex dynamic systems results inevitably in fuzziness, complexity and uncertainty. All these unfortunately complicate the problem of formal modeling the CDS and it supports the fact that complex dynamical systems are usually difficult to model, analyze, design, and optimally controlled [3],[4],[6],[7]. Thus the need for seeking new advanced conceptual modeling methods.

For all the above reasons the approach in modeling Complex Dynamic Systems using Fuzzy Cognitive Maps seem a promising as will be demonstrated in the next two sections.

\section{Basics of Fuzzy Cognitive Maps (FCM)}

Fuzzy Cognitive Maps (FCMs) is a new methodology for modeling complex dynamic systems and has been around only for the last 25-30 years. FCMs basically exploit the knowledge and experience of "people". Fuzzy Cognitive Maps came as a combination of the methods of fuzzy logic and neural networks. They constitute a computational method that is able to examine situations during which the human thinking process involves fuzzy or uncertain descriptions. Fuzzy Cognitive Maps were introduced by Kosko in 1988 [19] and they are a soft computing methodology that which gives users the ability to encounter problems in the same way the human mind does; using a conceptual procedure which can include ambiguous or fuzzy descriptions. They therefore offer an economical, flexible, fast and versatile approach to a variety of problems (social , political, economic , environmental and mechanical) which are extremely complex and a purely mathematical approach would be time consuming, laborious and require wasting many resources. Kosko introduced FCMs as a method to represent the causal relationship between concepts- nodes. Their goal is to represent knowledge in a symbolic way and model the behavior of systems containing elements with complex relationships, which sometimes can be hidden or illegible.

An FCM presents a graphical representation used to describe the cause and effect relations between nodes, thus giving us the opportunity to describe the behavior of a system in a simple and symbolic way. In order to ensure the operation of the system, FCMs embody the accumulated knowledge and experience from experts who know how the system behaves in different circumstances. In other words they recommend a modeling process consisting of an array of interconnected and interdependent nodes $\mathrm{Ci}$ (variables), as well as the relationships between them $\mathrm{W}$ (weights).Concepts take values in the interval $[0,1]$ and weights belong in the interval [-1,1]. A more comprehensive mathematical presentation of FCMs is provided in [14],[15],[16],[17],[18]. 


\section{An Illustrative Example When Using Fuzzy Cognitive Maps (FCM)}

With a simple example of Decision Making for the Stability of an Enterprise [8],[9],[10] in a Crisis Period using FCMs we can show that the new approach of FCMs in modelling CDS is very promising. In the current FCM model there is only one decision concept (output), i.e. the stability of an enterprise in a crisis period is studied: concept_8. The factor concepts are considered as measurements (via special statistic research) that determine how each measurement-concept will function in this model and they are: C1: sales, C2: turnover, C3: expenditures, C4: debts \& loans, C5: research \& innovation, C6: investments, C7: market share, C9: present capital, while C8: stability of enterprise is the output of the system.

Figure 1 shows a simple FCM model for the enterprise system. At this point it should be noted that in economic systems we can't talk about causality but only for correlation between the defined factorconcepts of this problem. Experts noted that the acceptable-desired region for the final value of concept C8 is:

$0.70 \leq C_{8}^{(\text {final })} \leq 0.95$

If C8(final) is inside this region then we can say with great certainty that the enterprise is out of danger and the economic crisis period does not put at risk the stability and the smooth function of the enterprise. Weights in table 1 are determined after defuzzifying (with COA method) the fuzzy values that were given from the experts (mostly economists).

Table 1. Weights between concepts for Enterprise System.

\begin{tabular}{|l|l|l|l|l|l|l|l|l|l|}
\hline & C1 & C2 & C3 & C4 & C5 & C6 & C7 & C8 & C9 \\
\hline C1 & 0 & 0.6 & 0 & -0.4 & 0.2 & 0.3 & 0.6 & 0.8 & 0 \\
\hline C2 & 0 & 0 & 0 & -0.2 & 0.2 & 0.5 & 0.1 & 0.3 & 0 \\
\hline C3 & 0 & 0 & 0 & 0.4 & -0.5 & -0.4 & 0 & -0.6 & -0.5 \\
\hline C4 & 0 & 0 & -0.4 & 0 & -0.7 & -0.8 & 0 & -0.7 & -0.4 \\
\hline C5 & 0.2 & 0.3 & 0 & 0 & 0 & 0.5 & 0.3 & 0.2 & -0.2 \\
\hline C6 & 0.3 & 0.2 & 0.6 & 0.5 & -0.3 & 0 & 0.3 & 0.3 & -0.4 \\
\hline C7 & 0.4 & 0.3 & 0 & -0.2 & 0 & 0 & 0 & 0.4 & 0.5 \\
\hline C8 & 0 & 0 & 0 & 0 & 0 & 0 & 0 & 0 & 0 \\
\hline C9 & 0 & 0 & 0 & -0.3 & 0.2 & 0.4 & 0 & 0.2 & 0 \\
\hline
\end{tabular}


Book of Proceedings

International Conference on Mechatronics, Sciences in Energy Efficiency Engineering,

System Engineering and Robotics

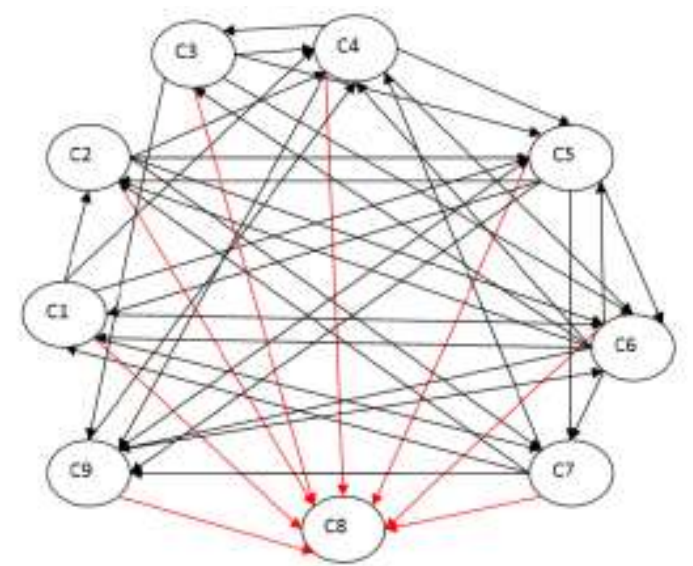

Fig. 1. A conceptual FCM model for Stability of the Enterprise.

In addition, the degree of occurrence of each input-concept factor is denoted with qualitative degrees of high, medium, and low. Respectively for the output concept C8 the qualitative degrees are very low, low, medium, high and very high.

Table 2. Initial factor-concepts fuzzy value.

\begin{tabular}{|l|l|}
\hline Factor-concepts & Case 1 \\
\hline C1 & $\mathrm{H}$ \\
\hline C2 & $\mathrm{M}$ \\
\hline C3 & $\mathrm{L}$ \\
\hline C4 & $\mathrm{L}$ \\
\hline C5 & $\mathrm{M}$ \\
\hline C6 & $\mathrm{L}$ \\
\hline C7 & $\mathrm{L}$ \\
\hline C9 & $\mathrm{M}$ \\
\hline
\end{tabular}

The initial values of the outputs were set equal to zero. The iterative procedure is being terminated when the values of $\mathrm{Ci}$ concepts has no difference between the latest three iterations. Considering $\lambda=1$ for the unipolar sigmoid function and after 11 iteration steps the FCM reaches an equilibrium point.

We considered initial values for the concepts: $A^{(0)}=\left[\begin{array}{llllll}0.8867 & 0.4667 & 0.0967 & 0.0967 & 0.4667 & 0.0967\end{array}\right.$ $0.09670 .650 .4667]$

It is observed that in the latest three iterations there is no difference between the values of concepts $\mathrm{Ci}$. So after 11 iteration steps, the FCM reaches an equilibrium point where the values do not change any more from their previous ones, that is:

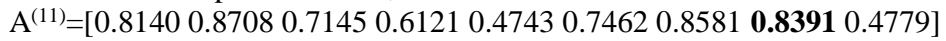

Decision concept C8 (Stability of the Enterprise) is equal to 0.8391 . 
Why Modeling Complex Dynamic Systems using Fuzzy Cognitive Maps?

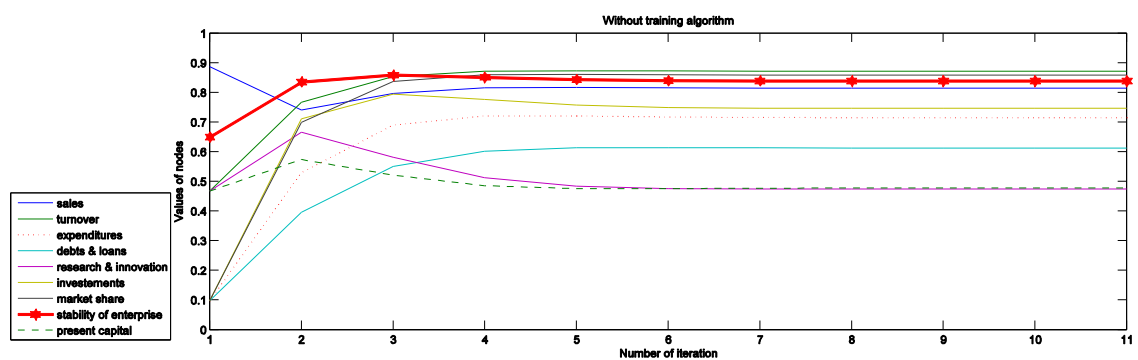

Fig. 2. Subsequent values of concepts till convergence.

Since the final value of C8(final) is inside the acceptable region, defined by the experts, then we could assume with great certainty that the enterprise can survive the crisis period.

\section{Conclusions and Future Research}

In this paper one of the most difficult and challenging problem in modelling, analyzing and controlling complex dynamic systems (CDS) has been seriously addressed. The analysis and efficient control of CDS are impossible without a formal model of the system. However today's' technologies for building such models for CDS are not sufficient. Qualitative description of most of the parameters of complex dynamic systems results inevitably in fuzziness, complexity and uncertainty. One of the challenges of accepting the "operation" of any complex dynamic system is the ability to make Decisions so the system runs efficiently and cost effectively. New conceptual and innovative approaches are needed. It is absolutely necessary to accept that Knowledge is the one and only one that can lead us in developing such models. And this knowledge must come from more than one expert who has extensive experience in observing and working on today's CDS. Decisions must be made by new Decision Making Support Systems (DMSS) which utilize new advanced and intelligent systems. Such a new approach is proposed to be Fuzzy Cognitive Maps (FCMs). FCMs offer the opportunity to produce better knowledge based on systems applications, addressing the need to handle uncertainties, fuzziness and inaccuracies associated with real CDS's problems. The illustrative example been provided in this plenary paper and the obtained results are promising for future research efforts in this exciting field of research.

Challenging future research directions include: new models of FCMs for CDS using learning methods; develop new DMSS using intelligent systems and advanced neural network theories; develop mathematical models using new advance FCMs for different applications and using a number of experts; develop new software tools for various CDS and perform extensive simulations.

\section{References}

1. Luenberger, D.G. "Introduction to dynamic systems: theory, models, and applications". Wiley, 1979.

2. Beltrami, E.J. "Mathematics for Dynamic Modeling", Vol 2. Academic Press,

3. Michel, A., Kaining W. Hu B. "Qualitative Theory of Dynamical Systems". Taylor \& Francis, 2001. 
Book of Proceedings International Conference on Mechatronics, Sciences in Energy Efficiency Engineering,

System Engineering and Robotics

4. Bar-Yam, Y. "Dynamics of complex systems". Reading, MA: Addison-Wesley, 1997.

5. Mesarovic, M., Macko, D. \& Takahara, Y. "Theory of hierarchical multilevel systems". New York: Academic Press, 1970.

6. Nelson, R.J. "Structure of Complex Systems". Philosophy of Science Association. 1976; 2:523542.

7. Keen, P.G. Morton, S. Michael, S. "Decision Support Systems: An organizational perspective". Addison-Wesley Pub. Co. (Reading, Mass.). 1978.

8. Ackoff R. L. "Management Misinformation Systems". Management Science. 1967; 14(4):147157.

9. Mintzberg, H. "Making Management Information Useful. Management Review”. 1975; 64(5): 34-38.

10. Gorry, G. A., Scott-Morton M. S. "A framework for management information systems". Sloan Management Review, 1971; 13(1):50-70.

11. Sprague, R. H. \& Watson, H. J. "Bit by Bit: Toward Decision Support Systems". California Management Review. 1979; 22(1): 60-68.

12. Gerrity, T. P. Jr. "Design of Man-Machine Decision Systems: An Application to Port- folio Management”. Sloan Management Review 12. 1971; 12(2):59-75.

13. Sprague Jr. R. H., \& Carlson. E. D. "Building effective decision support systems". Englewood Cliffs, NJ: Prentice-Hall, Inc. 1982.

14. Groumpos,P P., Stylios C.D. "Modeling supervisory control systems using fuzzy cognitive maps". Chaos Solitons \& Fractals. 2000; 113: 329-336.

15. Groumpos P.P. "Fuzzy Cognitive Maps: Basic Theories and their Application to Complex Systems". Glykas M. (ed) Fuzzy Cognitive Maps: Advances in Theory, Methodologies, Tools and Applications. Springer-Verlag Berlin, Heidelberg, 2010; 247: 1-22.

16. Papageorgiou E. I., Stylios C. D., Groumpos P. P.: "Active Hebbian learning algorithm to train fuzzy cognitive maps". International Journal of Approximate Reasoning, 2004;37: 219-249.

17. Groumpos P.P., Anninou A.P. “A theoretical mathematical modeling of Parkinson's disease using Fuzzy Cognitive Maps". 12th International Conference on Bioinformatics and Bioengineering (BIBE 2012), pp $677-682$.

18. Stylios, C.D., Groumpos, P.P. "Modeling complex systems using fuzzy cognitive maps". IEEE Transactions on Systems, Man and Cybernetics, Part A: Systems and Humans. 2004; 34(1):155162.

19. Kosko B. "Fuzzy cognitive maps". International Journal of man-machine studies. 1986; 24(1):6575 . 